\title{
Aportes al estudio del cortisol como marcador biológico del trastorno de personalidad antisocial
}

\author{
Soledad Sartori'1,2, Marcela López², María Lucía Zabala², Liliana Bakker ${ }^{2}$ \\ RESUMEN
}

\begin{abstract}
Objetivo: valorar en mujeres con diagnóstico de síndrome de Turner que presentaron trastorno de personalidad antisocial, niveles matutinos y vespertinos de cortisol y su pendiente circadiana, con el fin de contribuir a la discusión acerca de la vinculación entre trastornos de personalidad y secreción de cortisol. Material y métodos: se realizó un estudio de tipo exposfacto, retrospectivo, descriptivo, transversal con dos grupos. La muestra fue intencional conformada por mujeres con diagnóstico de síndrome de Turner que presentaron trastorno de personalidad antisocial y sus respectivos controles con edades comprendidas entre 18 a 40 años. Para evaluar trastornos de personalidad se utilizó el inventario clínico multiaxial de Millon II. Se determinó el cortisol libre en saliva, matutino y vespertino, para analizar funcionalidad del eje hipotálamo-hipófisis-suprarrenal. Resultados: se evidenciaron niveles inferiores de cortisol matutino y vespertino en participantes con diagnóstico de síndrome de Turner y existencia de diferencias estadísticamente significativas entre los resultados de la muestra clínica y control. Discusión: los resultados permiten observar que las mujeres con diagnóstico de síndrome de Turner presentaron trastorno de personalidad antisocial, exhiben una desregulación del eje hipotálamo-hipófisis-suprarrenal que se manifiesta en alteración del ritmo circadiano de cortisol con achatamiento de la pendiente diurna. Conclusiones: los datos obtenidos permiten enfatizar la importancia de profundizar en el estudio de las relaciones entre factores genéticos y ambientales, con su consecuente expresión en el sistema psiconeuroendócrino, en una población vulnerable como la abordada.
\end{abstract}

Palabras clave: cortisol, trastorno de personalidad antisocial, síndrome de Turner, mujeres.

\section{Contributions to the study of cortisol as a biomarker of the antisocial personality disorder}

\begin{abstract}
Aim: assess in women with diagnosis of Turner's syndrome who presented antisocial personality disorder, morning and evening levels of cortisol and its circadian slope, in order to contribute to the discussion about the relationship between personality disorders and cortisol secretion. Material and methods: we performed an ex post facto, retrospective, descriptive and cross-sectional study with two groups. The sample was intentional and conformed by women diagnosed with Turner's Syndrome who presented antisocial personality disorder and their respective control cases with ages between 18-40. To assess personality disorders we used the Millon Clinical Multiaxial Inventory II. Salivary free cortisol was determined, during morning and evening, to analyze the functionality of the hypothalamicpituitary-adrenal axis. Results: lower levels of morning and evening cortisol were evidenced in participants diagnosed with Turner's Syndrome so as the existence of statistically significant differences between the results of the clinical sample and the control sample. Discussion: results allow us to observe that women with diagnosis of Turner's Syndrome who presented antisocial personality disorders exhibit a deregulation of the hypothalamic-pituitary-adrenal axis, expressed in an alteration of the circadian rhythm of cortisol with flattening of the morning slope. Obtained data allow us to emphasize the importance of further study of the relationship between genetic and environmental factors and their subsequent expression in the psychoneuroendocrine system, in a vulnerable population as the addressed.
\end{abstract}


Key words: cortisol, antisocial personality disorder, Turner`s syndrome, women.

as neurociencias cognitivo sociales constituyen un nuevo campo disciplinar que intenta responder cuestiones fundamentales sobre la capacidad de las personas para entenderse; asimisimo, y a los demás, para desempeñarse efectivamente en el mundo social ${ }^{1}$. Vincula la investigación en psicología social y las neurociencias cognitivas, asumiendo como objetivo el estudio de las bases biológicas de la cognición y conductas sociales ${ }^{2}$, considerando que las habilidades cognitivas básicas contribuyen a la codificación, interpretación y acción efectiva sobre la realidad social le permiten a la persona un adecuado funcionamiento e inserción social.

En los últimos tiempos, el auge de esta disciplina ha abierto diversas líneas de investigación donde destacan estudios en teoría de la mente, empatía, funciones ejecutivas, memoria, atención y personalidad. Dentro de esta última temática los trastornos de la personalidad han adquirido gran relevancia, las investigaciones se han orientado al descubrimiento e identificación de marcadores biológicos que se asocian a los mismos. El cortisol ha sido una de las hormonas más estudiadas al respecto debido a la relación que diferentes investigaciones establecen entre la disminución en los niveles de secreción de esta hormona y conducta agresiva, la cual es un rasgo fundamental de algunos trastornos de personalidad ${ }^{3-10}$.

El cortisol es una hormona esteroidea o glucocorticoide, producida por la glándula suprarrenal en cuya regulación participa el eje neuroendócrino compuesto por hipotálamo-hipófisis-corteza de la glándula suprarrenal (HPA). En condiciones fisiológicas normales el cortisol muestra un marcado ritmo circadiano, con un incremento que oscila entre el 50 y $75 \%$ durante los primeros treinta minutos después del despertar, descendiendo con rápidez durante los siguientes treinta a sesenta minutos, para continuar su declinación de forma paulatina a lo largo del día, alcanzando sus niveles más bajos al final de la fase de actividad diurna ${ }^{11}$. En condiciones fisiológicas estables este ritmo ha presentado una significativa robustez, no afectándose por edad, género, etapa de desarrollo o composición corporal $^{12}$.

La desregulación del eje HPA; se manifiesta en alteración del ritmo circadiano, presentando cambios en el patrón de secreción matutino y vespertino, con ausencia o disminución de la pendiente de secreción. El achatamiento de la pendiente diurna de cortisol, producida por una baja tasa de declinación a lo largo del día, ha sido relacionado con estrés psicosocial agudo y estrés crónico; así como, con patologías subclínicas ${ }^{13}$.
Los resultados de las investigaciones realizadas en relación al vínculo entre secreción de cortisol y personalidad dan cuenta de diferencias en las evidencias reportadas, siendo hoy en día un tema controversial. Algunas de estas investigaciones indican que los rasgos o trastornos de personalidad juegan un papel relevante en las diferencias individuales de la respuesta endocrina del cortisol, tanto en sus niveles basales ${ }^{14}$ como ante estímulos estresantes ${ }^{15-17}$; mientras que otros trabajos $^{18-20}$ sostienen que no se han encontrado relaciones entre personalidad y patrón de respuesta fisiológica del cortisol.

Desde el modelo biosocial evolutivo de Millon el trastorno antisocial de la personalidad representa individuos que se caracterizan por tener una visión del mundo competitiva donde cada uno debe luchar por sí mismo mostrándose desconfiados y suspicaces, llegando en situaciones extremas a manifestar comportamientos ilegales tendientes a manipular el entorno a favor de sí mismos. Utilizan la agresividad como forma de defensa primaria, suelen actuar en forma impulsiva, por lo general carecen de habilidades empáticas, características que provocan dificultades sociales e interpersonales ${ }^{21}$.

La relación entre personalidad antisocial y niveles de cortisol ha sido descripta en varias investigaciones ${ }^{2232}$. Dentro de las anormalidades neuroendocrinológicas más reportadas en hombres antisociales se encuentra la disminución de los niveles de secreción de cortisol ${ }^{33-38}$. Al respecto Moss, Vanyukov y Martin ${ }^{39}$ realizaron un estudio de caso control comparando la secreción de cortisol ante situaciones de estrés en niños de entre 10 y 12 años con riesgo de presentar conducta agresiva y abusiva en la adolescencia a partir de su historia familiar. Los grupos se conformaron en relación a la presencia o no de historia familiar de agresión y abuso de sustancias, específicamente en los padres. Los resultados encontrados mostraron que los niños presentaban mayor riesgo de conducta antisocial, si sus padres tenían historial de abuso de sustancias y conducta violenta, tenían menores niveles de secreción de cortisol ante situaciones de estrés que los niños del grupo control

Recibido: 3 de agosto 2015. Aceptado: 25 agosto 2015.

${ }^{1}$ Consejo Nacional de Investigaciones Científicas y Técnicas (CONICET). ${ }^{2}$ Centro de Investigación en Procesos Básicos, Metodología y Educación (CIMEPB) Facultad de Psicología de la Universidad Nacional de Mar del Plata. Correspondencia: Soledad Sartori. Consejo Nacional de Investigaciones Científicas y Técnicas (CONICET). Funes 3250, Facultad de Psicología Nivel II. Mar del Plata (7600). Buenos Aires. Argentina. E-mail: solesartori@gmail.com 
cuyos padres no presentaban estas conductas negativas. Los investigadores sostienen que estos resultados podrían estar indicando que un menor aumento de los niveles de cortisol frente a situaciones de estrés podría ser un marcador biológico de futuros adolescentes antisociales. Asimismo, un estudio longitudinal con 38 niños en edad escolar que tenían síntomas de conducta agresiva (clínicamente reportada), a quienes se les valoró el ritmo circadiano de cortisol en saliva durante el segundo y el cuarto año de escolaridad; dio cuenta de la relación entre la disminución de la actividad del eje HPA y la presencia de conductas de agresión grave y persistente ${ }^{25}$.

De acuerdo a lo expuesto la relación entre personalidad antisocial y niveles de cortisol en el sexo masculino pareciera estar documentada por distintas investigaciones; sin embargo, esta asociación en el sexo femenino aún se encuentra en discusión. Algunos autores sostienen que, tal como sucede en los hombres, las mujeres con rasgos de personalidad antisocial presentan menores niveles de cortisol en saliva y sangre, mientras que otros autores argumentan que no existen suficientes evidencias empíricas que permitan confirmar esta asociación. Dentro del primer grupo una de las primeras investigaciones de relevancia fue la realizada por Susman, Schmeelk, Worrall, Granger, Ponirakis y Chrousos $^{29}$ quienes evaluaron los niveles de cortisol en 59 adolescentes embarazadas que presentaban síntomas de conducta antisocial. El análisis de los resultados mostró que una menor concentración de cortisol se encuentra asociada a un mayor número de signos de conducta antisocial. Después, Pajer, Gardner, Rubin, Perel y Neal ${ }^{40}$ investigaron los niveles matutinos de cortisol plasmático en una muestra de 84 mujeres adolescentes, 47 de las cuales cumplían con criterios para el diagnóstico de conducta antisocial y las 37 restantes fueron consideradas grupo control. Los resultados encontrados a partir de la comparación de los grupos indicaron que las mujeres adolescentes con conducta antisocial presentaban niveles más bajos de cortisol plasmático matutino, hecho que apoya la hipótesis que dichas mujeres podrían tener desregulación del eje HPA similar a la encontrada y reportada en los hombres antisociales.

Por otro lado, dentro del segundo grupo se encuentran, entre otros, Azar, Zoccolillo, Paquette, Quiros, Baltzer, y Tremblay ${ }^{41}$ quienes realizaron una investigación con 228 mujeres adolescentes embarazadas o en etapa de posparto, divididas en dos grupos a partir de la presencia o no de conducta antisocial, a todas ellas se les valoró la curva circadiana de cortisol en saliva; no encontrándose diferencias significativas entre los grupos.

La presente investigación intenta contribuir al de- bate presentado indagando en mujeres con diagnóstico de síndrome de Turner (ST) que presentan personalidad antisocial $^{42}$, los niveles matutinos y vespertinos de cortisol y su pendiente circadiana.

El ST es uno de los trastornos cromosómicos no heredables de mayor incidencia poblacional, 1:1900 nacidas vivas, que se encuentra determinado por deleción total o parcial del cromosoma $X$ en el sexo femenino ${ }^{43}$. Diversas investigaciones han correlacionado la variabilidad observada en el fenotipo con la variabilidad genotípica del síndrome, dando cuenta que la intensidad y frecuencia de las anomalías somáticas y de las características psicológicas están determinadas, en parte, por el grado de deleción y la inactivación o silenciamiento de genes. Sin embargo, estos mismos estudios, reportan diferencias individuales en mujeres con el mismo genotipo, aportando evidencias de la acción del ambiente en la expresión génica ${ }^{44}$. Entre sus principales características físicas se destacan baja talla y fallo en el desarrollo puberal. Estos problemas físicos, producen un impacto en el desarrollo psicológico y social de las mujeres con diagnóstico de ST y pueden afectar capacidad y calidad de sus relaciones sociales en especial en la adolescencia y adulta ${ }^{45-48}$. De esta forma las mujeres adultas con diagnóstico de ST presentan mayores dificultades para poder generar relaciones de amistad y pareja en parte debido al déficit que presentan en la comprensión de la comunicación no verbal y al sostenimiento de una imagen de sí mismas negativa, producto de las características físicas propias del síndrome ${ }^{49}$. Al mismo tiempo los estados de psicopatología como depresión, ansiedad y anorexia nerviosa son más frecuentes que en la población general ${ }^{50,51}$.

En relación a la personalidad, una investigación reciente -realizada en la ciudad de Mar del Plata, Buenos Aires, Argentina- muestra que dentro de la población de mujeres con diagnóstico de ST el trastorno antisocial de la personalidad obtiene un alto porcentaje de aparición. En la misma participaron 60 mujeres adultas con diagnóstico de ST a quienes se les administró el Inventario Clínico Multiaxial de Millon II para conocer sus características de personalidad y prevalencia de trastornos, encontrándose que aproximadamente el 30\% de las mismas presentaba trastorno antisocial de la personalidad solo o en combinación con otros trastornos ${ }^{42}$. Este porcentaje se considera elevado si se toman los datos del DSM V donde se sostiene que la prevalencia del trastorno antisocial de la personalidad en la población general de mujeres es del $1 \%^{52}$.

\section{OBJETIVO}

Valorar en las mujeres de la población citada que

Vol. 20 | No. 4 octubre-diciembre 2015 | 253 
presentaron con trastorno de personalidad antisocial, los niveles matutinos y vespertinos de cortisol y su pendiente circadiana, con el fin de contribuir a la discusión planteada acerca de la vinculación entre trastornos de personalidad y secreción de cortisol.

\section{MATERIAL Y MÉTODOS}

Se trabajó con un diseño de tipo exposfacto, retrospectivo, descriptivo, transversal y con dos grupos según la clasificación de Montero y León ${ }^{53}$, en una muestra de mujeres con diagnóstico de ST y sus respectivos controles.

La muestra fue intencional y corresponde a mujeres con diagnóstico de ST que presentan trastorno de personalidad antisocial $(N=17)$ y sus respectivos controles sin trastorno de personalidad antisocial con edades comprendidas entre los 18 a 40 años, para ambos grupos se les administró el Inventario Clinico Multiaxial de Millon II. Las participantes del grupo clínico fueron contactadas a través de convenios específicos establecidos con centros de salud referentes en la atención del síndrome de la ciudad de Mar del Plata y de la ciudad Autónoma de Buenos Aires. Por su parte las participantes incluidas en el grupo de control fueron contactadas a través de convenios con instituciones educativas terciarias, universitarias y grupos de trabajo lúdicos.

Se excluyeron mujeres con un índice de masa corporal mayor de $35 \mathrm{~kg} / \mathrm{m}^{2}$, diabetes, insuficiencia hepática o renal crónica, tratamiento con corticoides en el último año y mujeres que hayan cursado alguna enfermedad aguda en las últimas dos semanas.

En una primera etapa se estableció contacto con mujeres con diagnóstico de ST que habían participado de una investigación previa de personalidad realizada por el grupo de investigación, que presentaban trastorno de personalidad antisocial. Se les explicó el objetivo y características de esta nueva investigación, solicitándoles firma del consentimiento informado, aclarando expresamente que la colaboración era voluntaria y anónima. Simultáneamente se seleccionó una posible población control, explicándoles los alcances de la investigación y solicitándoles la firma del consentimiento informado. Después se le administró a la población control seleccionada el Inventario Clínico Multiaxial de Millon II. A partir de los resultados obtenidos en el cuestionario, se seleccionó la población control definitiva (mujeres adultas sin trastorno de personalidad antisocial).

Finalizada esta primera etapa, a cada participante se le entregó el protocolo de extracción de la muestra salival, indicándoles no cepillarse los dientes antes de recolectar la muestra para evitar el sangrado de las encías, no ingerir agua o alimentos, ni utilizar inhaladores que contengan esteroides. Se obtuvo en promedio $1 \mathrm{ml}$ de saliva, recolectada por salivación directa dentro de un tubo de vidrio estéril sin aditivos, que se almacenó a $4^{\circ} \mathrm{C}$. Se realizaron dos tomas diarias, inmediatamente después de levantarse (matutino: 8:00 hs) y a media tarde (vespertino: 18:00 hs).

Para la evaluación de los trastornos de personalidad se utilizó el Inventario Clínico Multiaxial de Millon II (MCMI-II) que evalúa estilos básicos de funcionamiento de la personalidad sostenidos por Millon y definidos según las denominaciones del eje II del DSM- IV; junto con los síndromes clínicos del eje I del citado manual diagnóstico. El inventario está conformado por 175 ítems con formato de respuesta dicotómico (verdadero- falso) distribuidos en 26 escalas que recogen diferentes aspectos de la personalidad patológica: 4 escalas de fiabilidad y validez, 10 escalas básicas de personalidad (esquizoide, fóbica, dependiente, histriónica, narcisista, antisocial, agresivo-sádica, compulsiva, pasivo-agresiva y autodestruc-tiva), 3 de personalidad patológica (esquizoide, límite y paranoide), 6 síndromes clínicos de gravedad moderada (ansiedad, histeriforme, hipomanía, distimia, abuso de alcohol y abuso de drogas) y 3 síndromes clínicos de gravedad severa (pensamiento psicótico, depresión mayor y trastorno delirante).

Por otro lado, se determinó el cortisol libre en saliva, matutino (8:00 hs) y vespertino (18:00 hs), el cual ofrece ventajas sobre otras mediciones como sangre $u$ orina. El método de recolección es indoloro y no invasivo, reduciendo el estrés que produce extracción y evitando el sesgo que pudiera producirse a raíz del procedimiento. Se ha comprobado que las cifras de cortisol salival representan de manera adecuada a las cifras plasmá-ticas (sangre), siendo una medida que se mantiene estable por varias semanas y permite cuantificar de forma exacta y eficiente el cortisol biológicamente $\operatorname{activo}^{54}$.

El cortisol salival se midió por radioinmunoanálisis competitivo, utilizando el kit comercial Coat-acount con estándares diluidos 1/10. La sensibilidad analítica del método corresponde a $8.28 \mathrm{nmol} / \mathrm{l}$ y las variaciones intra e interensayo se encuentran por debajo del $6.1 \mathrm{y}$ 7.1\% respectivamente. El estudio se realizó en el Laboratorio de Análisis Clínico y Endocrinológico Laycet (LACYE 90240) de la ciudad de Mar del Plata (Argentina); acreditado por la Fundación Bioquímica Argentina CONEA con evaluación de calidad y permiso para el uso de trazadores radiactivos in vitro en humanos.

Para analizar la pendiente circadiana de cortisol se utilizó el protocolo de Adam y Kumari ${ }^{13}$, quienes proponen la obtención de un coeficiente que refleja la declinación diaria del cortisol. El mismo se obtiene restándole al valor vespertino de cortisol el valor matutino 
y dividiendo el número resultante por la cantidad de horas que separan ambas muestras.

Para contrastar los valores matutinos y vespertinos de cortisol y la pendiente circadiana de cortisol se realizaron análisis descriptivos e inferenciales no paramétricos (debido al tamaño pequeño de la muestra) de diferencia de medias para dos muestras independientes ( $U$ de Mann-Whitney), valorando el tamaño del efecto de las diferencias ( $d^{\prime}$ de Cohen).

\section{Consideraciones éticas}

La participación fue voluntaria, sujeta al consentimiento informado y asentimiento de las participantes. Durante el desarrollo del trabajo se respetaron principios éticos de la investigación con seres humanos, procurándose las condiciones necesarias para proteger la confidencialidad y actuar en beneficio de las participantes. El protocolo de investigación fue aprobado por el Comité de Ética del Consejo Institucional de Revisión de Estudios (CIREI) y el Comité de Ética de la Facultad de Psicología de la Universidad Nacional de Mar del Plata (UNMDP).

\section{RESULTADOS}

En la tabla 1, se presentan las medidas de tendencia central y variabilidad de los valores de cortisol salival matutinos y vespertinos, conjuntamente con el tamaño del efecto. La tabla 2 exhibe los resultados del análisis no paramétrico de comparación de medias para dos muestras independientes.

Los resultados presentados en las tablas 1 y 2 evidencian niveles inferiores de cortisol matutinos y vespertinos en las participantes con diagnóstico de ST y la existencia de diferencias estadísticamente significativas entre los resultados de las participantes de la muestra clínica y sus controles en ambos índices, con tamaños del efecto de las diferencias altos.

Siguiendo con el análisis de la pendiente de cortisol se aplicaron estadísticas descriptivas e inferenciales a los datos del coeficiente de declinación

Tabla 1. Estadísticos descriptivos de los índices correspondientes a los valores de cortisol (nmol/l) matutino y vespertino para el grupo clínico y control.

\begin{tabular}{cccc}
\hline Índices & $\begin{array}{c}\text { Clínico } \\
(n=17)\end{array}$ & $\begin{array}{c}\text { Control } \\
(n=17)\end{array}$ & Cohen's d \\
& $M(D S)$ & $M(D S)$ & \\
\hline Cortisol matutino & $17,72(6,31)$ & $24,03(8,72)$ & $-0,83$ \\
Cortisol vespertino & $13,52(7,72)$ & $9,24(2,42)$ & 0,75 \\
\hline
\end{tabular}

propuesto por Adam y Kumari ${ }^{13}$, los resultados se presentan en las tablas 3 y 4.

Tabla 2. Diferencias en los índices correspondientes a los valores de cortisol ( $\mathrm{nmol} / \mathrm{I})$ matutino y vespertino entre grupo clínico y control.

\begin{tabular}{ccc}
\hline Índices & U Mann-Whitney & $\mathrm{Z}$ \\
\hline Cortisol matutino & $98,00^{*}$ & $-2,19$ \\
Cortisol vespertino & $107,50 *$ & $-1,91$ \\
\hline${ }^{* *} p<.01$ - $^{*} p<.05$ & &
\end{tabular}

Tabla 3. Estadísticos descriptivos del coeficiente de declinación del cortisol (nmol/l), para el grupo clínico y el grupo control.

\begin{tabular}{cccc}
\hline Índice & Clínico & Control & Cohen's d \\
& $(n=17)$ & $(n=17)$ & \\
$M(D S)$ & $M(D S)$ & \\
Coeficiente de declinación & $-0,82(0,47)$ & $-1,26(0,68)$ & $-0,75$ \\
\hline
\end{tabular}

Tabla 4. Diferencias en el índice correspondiente al coeficiente de declinación del cortisol ( $\mathrm{nmol} / \mathrm{l})$, para el grupo clínico y control.

\begin{tabular}{ccc}
\hline \multicolumn{1}{c}{ Índice } & U Mann-Whitney & $\mathrm{Z}$ \\
\hline Coeficiente de declinación & $107,00^{*}$ & $-1,92$ \\
\hline${ }^{* *} p<.01-{ }^{*} p<.05$ & &
\end{tabular}

Los resultados muestran un nivel de significancia inferior a 0,05 rechazándose la hipótesis de igualdad de medias, resultando que las diferencias halladas son estadísticamente significativas.

\section{DISCUSIÓN}

Los resultados, si bien limitados debido al tamaño de la muestra, permiten observar que las mujeres con diagnóstico de ST que presentaron trastorno de personalidad antisocial exhiben una desregulación del eje HPA que se manifiesta en una alteración del ritmo circadiano de cortisol con achatamiento de la pendiente diurna. La estructura de la pendiente está dada por bajas concentraciones de cortisol matutino y vespertino en comparación con su grupo control.

Estos datos plantean correspondencia con líneas de investigación desarrolladas por Susman ${ }^{29}$, Pajer ${ }^{40}$ y McBurnett, Lahey, Rathouz y Loeber ${ }^{50}$ las cuales sostienen que la personalidad antisocial se vincula con un descenso de los niveles matutinos y vespertinos de cortisol en sangre y saliva; así como, menor reactividad del eje HPA ante situaciones de estrés.

Sin embargo, para poder concluir que las caracte-

Vol. 20 | No. 4 octubre-diciembre 2015 | 255 
rísticas observadas en el ritmo circadiano de cortisol de las mujeres con diagnóstico de ST evaluadas, podrían constituirse en un marcador biológico de personalidad antisocial, sería conveniente ampliar el estudio a mujeres con diagnóstico de ST, sin trastorno de personalidad antisocial, para descartar que la alteración del ritmo fuera inherente al síndrome.

En este punto es importante considerar que numerosas investigaciones reportan en mujeres con este diagnóstico la existencia de alteraciones anatómicas en la amígdala e hipotálamo. McCauley y Sybert ${ }^{47}$ y Kesler ${ }^{55}$ han observado un agrandamiento en el volumen amigdalino izquierdo que involucra sustancia gris y una reducción del hipocampo derecho incluyendo sustancia gris y blanca. Estas áreas cerebrales participan en la regulación del ritmo circadiano de cortisol. En consecuencia, considerando que la alteración anatómica y funcional del hipocampo y amígdala es un defecto primario del desarrollo neural de las mujeres con diagnóstico de ST, podría pensarse que estas alteraciones harían más vulnerables a las mujeres con diagnóstico de ST de padecer trastorno de personalidad antisocial, consecuencia de la alteración de su ritmo de cortisol.

\section{CONCLUSIÓN}

La presente investigación se constituye en un inicio de trabajo en esta área; sin embargo, los datos obtenidos permiten pensar en la importancia de ahondar en el estudio de las relaciones entre factores genéticos y ambientales, con su consecuente expresión en el sistema psiconeuroendócrino, en una población vulnerable como la abordada. Asimismo, es importante considerar la acción del ambiente como modulador de las características de personalidad y la posibilidad de establecer acciones asociadas a los vínculos, de la educación y socialización que busquen prevenir la aparición del trastorno de personalidad o disminuir las características desadaptativas alcanzando un mejor ajuste al medio social.

\section{REFERENCIAS}

1. Ochsner K, Lieberman M. The emergence of social cognitive neuroscience. Am Psychol 2001; 56(9):717-34.

2. Grande García I. Neurociencia social: el maridaje entre la psicología social y las neurociencias cognitivas. Revisión e introducción a una nueva disciplina. Anal Psicol 2009;25(1): 1-20.

3. Alink LRA, van ljzendoorn $\mathrm{MH}$, Bakermans Kranenburg $\mathrm{MJ}$, Mesman J. Cortisol and externalizing behavior in children and adolescents: mixed meta-analytic evidence for the inverse relation of basal cortisol and cortisol reactivity with externalizing behavior. Dev Psychobiol 2008; 50(5):427-50.

4. Carré JM, Mehta PH. Importance of considering testosterone- cortisol interactions in predicting human aggression and dominance. Aggressive Behav 2011; 37(6):489-91.

5. Feilhauer J, Cima M, Korebrits A, Nicholson NA. Salivary cortisol and psychopathy dimensions in detained antisocial adolescents. Psychoneuroendocrinology 2013;38(9):1586-95.

6. van Goozen SH, Matthys W, Cohen Kettenis PT, Buitelaar JK, van Engeland $\mathrm{H}$. Hypothalamic-pituitary-adrenal axis and autonomic nervous system activity in disruptive children and matched controls. J Am Acad Child Adolesc Psychiatry 2000; 39(11):1438-45.

7. Gowin JL, Green CE, Alcorn JL, Swann AC. The role of cortisol and psychopathy in the cycle of violence. Psychopharmacology (Berl) 2013; 227(4):661-72.

8. Hawes DJ, Brennan J, Dadds MR. Cortisol, callous- unemotional traits, and pathways to antisocial behavior. Curr Opin Psychiatr 2009; 22(4):357-62.

9. O'Leary MM, Taylor J, Eckel L. Psychopathic personality traits and cortisol response to stress: the role of sex, type of stressor, and menstrual phase. Horm Behav 2010;58(2):250-6.

10. Shirtcliff EA, Vitacco MJ, Graf AR, Gostisha AJ. Neurobiology of empathy and callousness: implications for the development of antisocial behavior. Behav Sci Law 2009; 27(2):137-71.

11. Ranjit A, Young EA, Raghunathan TE, Kaplan GA. Modeling cortisol rhythms in population-based study. Psichoneuroendocrinology 2005;30:615-24.

12. Rosmalen JGM, Oldehinkel AJ, Ormel J, de Winter AF, Buitelaar JK. Determinants of salivary cortisol levels in 10-12 year old children; a population-based study of individual differences. Psychoneuroendocrinology 2005; 30:483-95.

13. Adam EK, Kumari M. Assessing salivary cortisol in largescale, epidemiological research. Psychoneuroendocrinology 2009; 34:1423-36.

14. Kirschbaum C, Bartussek D, Strasburger CJ. Cortisol responses to psychosocial stress and correlations with personality traits. Pers Indiv Differ 1992;13(12):1353-7.

15. al'Absi M, Bongard S, Lovallor WR. Adrenocorticotropin responses to interpersonal stress: effects of overt anger expression style and defensiveness. Int J Psychophysiol 2000; 37:257-65.

16. Pérez-García AM, Sanjuán P, Bermúdez J. Control personal y situacional y reactividad cardiovascular en tareas de estrés físico. Psicothema 2000;14(3):583-90.

17. Senior C, Loveday C. A pilot investigation into the neuroendocrine correlations of personality in the workplace. Percept Mot Skills 1998;87:794.

18. Bossert S, Berger M, Krieg JC, Schreiber W, Junker M. Cortisol response to various stressful situations: relationship to personality variables and coping styles. Neuropsychobiology 1988;20(1):36-42.

19. Adler LW, Wedekind D, Pilz J, Weniger G, Huether G. Endocrine correlates of personality traits: a comparison between emotionally stable and emotionally labile healthy young men. Neuropsychobiology 1997;35:205-10.

20. Schommer NC, Kudielka BM, Hellhammer D, Kirschbaum C. No evidence for a close relationship between personality traits and circadian cortisol rhythm or a single cortisol stress response. Psychol Rep 1999;84:840-2.

21. Choca JP, Van Denburg E. Guía para la interpretación del MCMI. Buenos Aires: Editorial Paidós. 1998.

22. Cima M, Smeets T, Jelicic M. Self-reported trauma, cortisol levels, and aggression in psychopathic and non-psychopathic prison inmates. Biol Psychol 2008;78(1):75-86.

23. Fries E, Dettenborn L, Kirschbaum C. The cortisol awakening response: facts and future directions. Int J Psychophysiol 2009; 72(1):67-73.

24. Heim C, Ehlert U, Hellhammer DH. The potential role of 
hypocortisolism in the pathology of stressrelated bodily disorders. Psychoneuroendocrinology 2000;25(1):1-35.

25. McBurnett K, Lahey BB, Rathouz PJ, Loeber R. Low salivary control and persistent aggression in boys referred for disruptive behavior. Arch Gen Psychiatry 2000;57(1):38-43.

26. Moss HB, Vanyukov MM, Yao JK, Kirillova GP. Salivary cortisol responses in the prepuberal boys: the effects of parental substance abuse and association with drug use behavior during adolescence. Biol Psychiatry 1999;45(10):1293-9.

27. O'Leary MM, Loney BR, Eckel LA. Gender differences in the association between psychopathic personality traits and cortisol response to induced stress. Psychoneuroendocrinology 2007;32(2):183-91.

28. Schulz KP, Halperin JM, Newcorn JH, Sharma V. Plasma cortisol and aggression in boys with ADHD. J Am Acad Child Adolesc Psychiatry 1997;36(5):605-9.

29. Susman EJ, Schmeelk KH, Worrall BK, Granger DA. Corticotropin releasing hormone and cortisol: longitudinal associations with depression and antisocial behavior in pregnant adolescents. J Am Acad Child Adolesc Psychiatry 1999;38(4):460-7.

30. van Goozen S, Matthys W, Cohen Kettenis P, Gispen de Wied $C$, et al. Salivary cortisol and cardiovascular activity during stress in oppositional-defiant disorder boys and normal controls. Biol Psychiatry 1998;43: 531-9.

31. Vanyukov M, Moss H, Plail J, Blackson T, Mezzich A, et al. Antisocial symptoms in preadolescent boys and in their parents: associations with cortisol. Psychiatry Res 1993;46:9-17.

32. Welker KM, Lozoya E, Campbell JA, Neumann CS. Testosterone, cortisol and psychopathic traits in men and women. Physiol Behav 2014:129:230-6.

33. Dolan M, Anderson IM, Deakin JF. Relationship between 5-HT function and impulsivity and aggression in male offenders with personality disorders. Br J Psychiatry 2001;178(4):352-9.

34. Johnson MM, Caron KM, Mikolajewski AJ, Shirtcliff EA. Phychopathic traita, empathy and aggression are differentially related to cortisol awakening reponse. J Psychopathol Behav Assess 2014;36:380-8.

35. McBurnett K, Lahey BB, Capasso L, Loeber R. Aggresive symptoms and salivary cortisol in clinic-referred boys with conduct disorder. Ann N Y Acad Sci 1996; 794(1):169-78.

36. Murray Close D, Han G, Cicchetti D, Crick NR. Neuroendocrine regulation and physical and relational aggression: the moderating role of child maltreatment and gender. Dev Psychol 2008; 44(4):1160-76.

37. von Polier GG. Reduce cortisol in boys with early-onset conduct disorder and callous-unemotional traits. BioMed Res Int 2013;1-9.

38. Shoal GD, Giancola PR, Kirillova GP. Salivary cortisol, personality and aggressive behavior in adolescent boys: a 5Year longitudinal study. J Am Acad Child Adolesc Psychiatry 2003;42(9):1101-7.
39. Moss HB, Vanyukov MM, Martin CS. Salivary cortisol responses and the risk for substance abuse in prepuberal boys. Biol Psychiatry 1995; 38(8):547-55.

40. Pajer K, Gardner W, Rubin RT, Perel J. Decreased cortial levels in adolescent girls with conduct disorder. Arch Gen Psychiatry 2001;58(3):297-302.

41. Azar R, Z occolillo M, Paquette D, Quiros E, Baltzer F, Tremblay RE. Cortisol levels and conduct disorder in adolescent mothers. J Am Acad Child Adolesc Psychiatry 2004;43(4):461-8.

42. Sartori MS, Urquijo S, López M, Said A. Análisis de perfiles de personalidad en mujeres adultas con diagnóstico de síndrome de Turner. Interdisciplinaria En Prensa.

43. Murphy M, Mazzocco M, Gerner G, Henry A. Mathematics learning disability in girls with Turner syndrome or fragile $X$ syndrome. Brain Cogn 2006;61(2):195-210.

44. López MC, Aguilar MJ. Vulnerabilidad social en el Síndrome de Turner: interacción genes- ambiente. Psicología: ciencia y profesión 2009; 29(2):318-29.

45. Aguilar MJ, López MC, Sartori S. Autoconcepto y Síndrome de Turner: influencia de parámetros biológicos en el desarrollo psicosocial. Ac Psiquiátr Psicol Am Lat 2010;56(4):274-83.

46. El Abd S, Turk J, Hill P. Annotation: Psychological characteristics of Turner syndrome. J Child Psychol Psychiatry 1995;36(7):1109-25.

47. McCauley E, Sybert V. Social and behavioral development of girls and women with Turner syndrome. International Congress Series 2006;1298:93-9.

48. Suzigan LZ, Paiva e Silva RB, Guerra Júnior G, Mariani SH, Maciel Guerra AT. Social skills in women with Turner Syndrome. Scand J Psychol 2011;52(5):440-7.

49. Elsheikh M, Dunger DB, Conway GS, Wass JA. Turner's syndrome in adulthood. Endocr Rev 2002;23(1):120-40.

50. Delooz J, Van den Berghe H, Swillen A, Kleczkowska A. Turner syndrome patients as adults: a study of their cognitive profile, psychosocial functioning and psychopatho-logical findings. Genet Couns 1993;4(3):169-79.

51. Labarta Aizpún J. Síndrome de Turner: crecimiento espontáneo y talla final. En Sociedad Española de Endocrinología Pediátrica (SEEP) (Eds.), Síndrome de Turner: $9^{\circ}$ curso de formación de posgrado. 2004; 51-70.

52. Asociación Americana de Psiquiatría. Guía de Consulta de los criterios diagnósticos del DSM 5. 2013. Estados Unidos: Asociación Americana de Psiquiatría.

53. Montero I, León O. A guide for naming research studies in Psychology. Int J Clin Health Psychol 2007; 7(3):847-62.

54. Mirasoli M, Deo SK, Lewis JC, Roda A. Bioluminescence inmunoassay for cortisol using recombinant aequorin as a label. Anal Biochem 2002; 306(2):204-11.

55. Kesler S, Garrett A, Bender B, Yankowitz J, Zeng SM. Amygdala and hippocampal volumes in Turner syndrome: a highresolution MRI study of X-monosomy. Neuropsychologia 2004; 42:1971-8.

\section{ARTÍCULO SIN CONFLICTO DE INTERÉS}

